\title{
Inhibitory avoidance learning altered ensemble activity of amygdaloid neurons in rats
}

\author{
Chun-Hui Chang, ${ }^{1}$ Keng-Chen Liang ${ }^{2}$ and Chen-Tung Yen ${ }^{1,3}$ \\ ${ }_{1}^{1}$ Institute of Zoology, National Taiwan University, No. 1, Roosevelt Road, Sec. 4, Taipei 106, Taiwan \\ ${ }^{2}$ Department of Psychology, National Taiwan University, Taipei, Taiwan \\ ${ }^{3}$ Department of Life Science, National Taiwan University, Taipei, Taiwan
}

Keywords: amygdala, conditioning, fear, habituation, memory, single-unit activity

\begin{abstract}
In this study, we examined single-unit activity in the amygdala before and after a rat had acquired an inhibitory avoidance task. LongEvans rats with microwires chronically implanted into the central nucleus $(\mathrm{CeA})$ or basolateral complex $(\mathrm{BLC})$ of the amygdala were acclimatized to the apparatus of a step-through inhibitory avoidance task for three sessions. On the fourth session, rats in the experimental group received an inescapable footshock $(3 \mathrm{~mA}, 1 \mathrm{~s})$ as they stepped from the lit side into the dark side of the task apparatus, whereas rats in the control group received the same amount of shock on a different apparatus. All rats were tested for retention in the task apparatus 1 day after shock training. The experimental rats showed better retention than the controls as they stayed longer in the lit side. Ensemble unit activities were recorded in the amygdala nuclei from the indwelling wire bundles during the acclimation and test sessions. The data collected from well-isolated amygdala units showed that neuronal discharge habituated from the first to the third acclimation session. In the test session, the experimental group, but not the control group, showed elevated firing rates in the $\mathrm{CeA}$ or BLC neurons located on either side of the brain. These findings provide the first piece of evidence showing that learning of an inhibitory avoidance task leads to an increase in amygdala neuronal discharges during a retention test.
\end{abstract}

\section{Introduction}

Memory of a fearful experience is unique for its rapid acquisition and long-lasting retention. Therefore, learning paradigms motivated by fear are often used to study the neural mechanism underlying memory (Cahill \& McGaugh, 1998; LeDoux, 2000). In classical fear conditioning, a neutral stimulus is followed by an aversive event and then fear responses, such as changes in the heart rate and blood pressure, freezing, or acoustic startle responses, are monitored as indices of memory when the conditioned stimulus is re-encountered in a retention test (Pascoe \& Kapp, 1985; Repa et al., 2001; Walker \& Davis, 2002; Goosens et al., 2003). Memory of a fearful experience can also be assessed by operant conditioning in which animals learn to escape from or avoid a discriminative cue signalling an aversive consequence in a specific context (McGaugh et al., 2000; Poremba \& Gabriel, 2001).

Extensive evidence implicates the amygdala in memory processing of affective-laden information (Aggleton, 2000). Evidence has shown that lesions or electrical stimulation of the amygdala before or after training impaired acquisition and/or retention in various aversively motivated learning tasks (LeDoux, 2000; Maren, 2001). Studies have also shown that a post-training intra-amygdala infusion of pharmacological agents, such as norepinephrine or other neurochemicals, alters retention in one-trial inhibitory avoidance tasks (Liang et al., 1986, 1990, 1994, 1996). These data have been interpreted as either plasticity in the amygdala subserving the neural trace of the fear memory (Fanselow \& LeDoux, 1999) or the amygdala modulating

Correspondence: Professor Chen-Tung Yen, ${ }^{1}$ Institute of Zoology, as above. E-mail: ctyen@ntu.edu.tw

Received 8 June 2004, revised 14 October 2004, accepted 18 October 2004 memory of the experience formed elsewhere in the brain (McGaugh, 2004; Roozendaal et al., 2004).

To understand the exact nature of amygdaloid functions in affective memory, direct recording of neuronal activity from the nuclei is helpful. Previous evidence has shown that classical fear conditioning indeed alters neuronal activity within the amygdala (LeDoux, 2000). In rabbits, unit firing in the central nucleus of the amygdala (CeA) changed in parallel with growth of the conditioned response during Pavlovian heart rate conditioning (Pascoe \& Kapp, 1985). In rats, fear conditioning also increases unit firing in the lateral amygdala nucleus in response to a tone serving as the conditioned stimulus (Quirk et al., 1995, 1997; Repa et al., 2001; Goosens et al., 2003). Additionally, activity changes in amygdaloid neurons have also been reported in an early phase of active avoidance learning in rabbits (Maren et al., 1991).

The inhibitory avoidance task is a widely adopted task in assessing the role of the amygdala in learning and memory. However, no extant study, to the best of our knowledge, has formally addressed the questions of whether and how learning such a task may change neuronal activity in the amygdala. In an attempt to elucidate these issues, the present study was designed to record single-unit activities from the CeA and basolateral complex (BLC; including the basolateral and lateral amygdala nuclei) of rats at various phases of the stepthrough inhibitory avoidance task.

\section{Materials and methods}

\section{Subjects}

The subjects were 16 female Long-Evans rats purchased from the National Laboratory Animal Center (Nankang, Taipei, Taiwan) at the 
age of 4-6 weeks old. After arriving from the breeding centre, they were housed in groups of three or four per cage in the animal room of Life Science Building, National Taiwan University, Taipei. Food and water were available ad libitum. Surgery was performed at least 1 month after arrival at which time the animal weighed $250-350 \mathrm{~g}$. At least 1 week before the surgery, animals were moved to new individual cages. The care of the animals and the entire experimental procedure were in accordance with Codes for Experimental Use of Animals based on Animal Protection Law of the Council of Agriculture, Taiwan, and were approved by the Institutional Animal Care and Use Committee of National Taiwan University.

\section{Implantation of recording electrodes}

A bundle-type electrode consisting of eight microwires (no. 15140/13848, $50 \mu \mathrm{m}$ in diameter; California Fine Wire Co., USA) was used. These electrodes were built in-house according to procedures modified from a previous study (Tsai \& Yen, 2003) and cut to a length $(\approx 12 \mathrm{~mm})$ appropriate for recording the unit activity in the amygdala.

To implant the electrode, subjects were pretreated with atropine $(0.24 \mathrm{mg} / \mathrm{kg}$, intraperitoneally) and were anaesthetized with sodium pentobarbital (50 mg/kg, intraperitoneally). The surgery commenced only when the rat was fully anaesthetized and had totally lost the withdrawal reflex to a pinch. Throughout the surgery, the anaesthetized state was carefully monitored and supplemental doses of the same anaesthetic were administered whenever needed. The body temperature was maintained at $37.5^{\circ} \mathrm{C}$ by a heating pad. The animal was mounted on a stereotaxic instrument and the recording electrodes were implanted into the right $(n=2)$ or both sides $(n=14)$ of the amygdala, aiming at the CeA or BLC. The coordinates for the targets were AP, -2.0 to $-3.3 \mathrm{~mm}, \mathrm{ML}, \pm 4.5-5.2 \mathrm{~mm}$ and $\mathrm{DV},-8.0$ to $-8.2 \mathrm{~mm}$ according to the atlas of Paxinos \& Watson (1999). Burr holes were drilled into the skull over the target regions and recording electrodes were lowered into the target areas. Four stainless steel screws were implanted onto the skull as anchors. These screws were connected with a stainless steel wire serving as the electric ground. All wires were plugged into a headpiece connector and the entire assembly was affixed onto the skull with dental cement. The animal was allowed 7-10 days of recuperation before being subjected to any behavioural and electrophysiological experiments.

\section{Neuronal data collection}

To record electrical activity, an adaptor connected to a recording cable was plugged into the headpiece on the rat. The recorded signals were first fed into a unity-gain operational amplifiers through a preamplifier (Plexon PBX/32HP; Dallas, TX, USA) and then into a 32-channel multiple-channel acquisition processor (MAP) system (Plexon). Highfrequency signals were selected with a band-pass filter set at $0.5-8.8$ or $13 \mathrm{kHz}$. After entering the MAP system, wavelets $>1.5 \times$ the peakto-peak noise level were selected and saved using the software SortClient (Plexon). At a maximal background noise of $\approx 60 \mu \mathrm{V}$, the signal-to-noise ratio of selected neurons fell between 1.6 and 4.8 with a median of 2.4. The data files were then imported into the software Offline Sorter (Plexon) which isolated single units on each channel using principal component analyses and a template-matching algorithm. Inter-spike interval histograms of each sorted neuron were examined to ensure that firing events were separated by $>1 \mathrm{~ms}$ (Setlow et al., 2003), which served as a way of validating that these waveforms were generated by an individual neuron. An example of

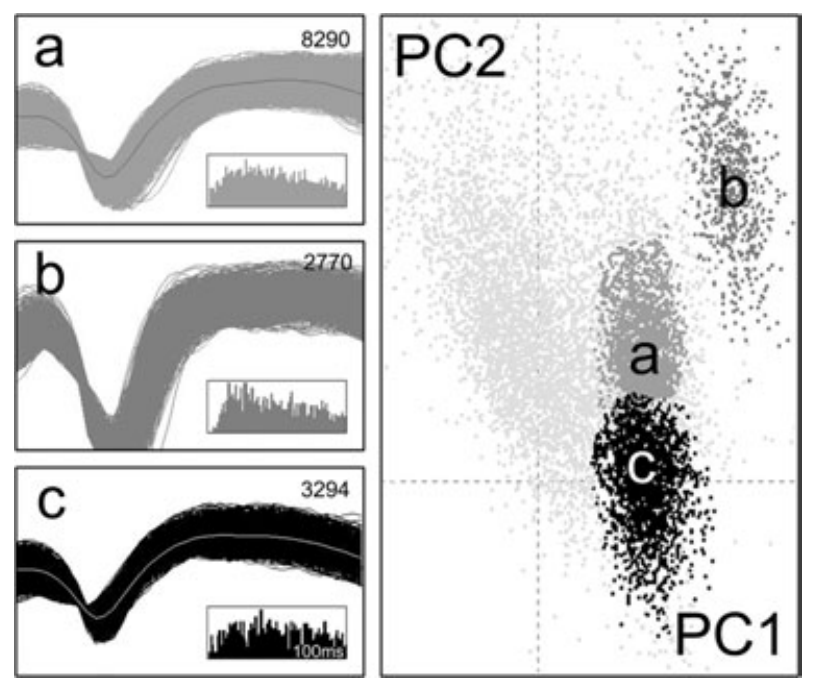

FIG. 1. Cumulative waveforms (left panel), interspike interval histograms (insets), and the principal component plot (right panel) of three sorted units (units $\mathrm{a}, \mathrm{b}$, and c) from one recording microwire. The number of spikes of the three units during the 930-s recording period is shown in the top right corner in each waveform figure. Segments of the spike waveforms (1-ms) are shown in the left panels. The bin width in the 100-ms interspike interval histogram is $1 \mathrm{~ms}$.

the sorted single units from one recording channel is shown in Fig. 1. Sorted files and single-unit firing rates were then analysed using the software Nex (Plexon).

\section{Inhibitory avoidance learning}

All rats received training and testing on an inhibitory avoidance task in a quiet, dimly lit room, in order to minimize interference with the learning process. Throughout experiments, behaviour was taped with a video camera with a night shot mode for further analysis. Video frames and the electrical recording file were synchronized with the aid of a video timer.

The apparatus was a trough-shaped alley $(95 \mathrm{~cm}$ long, $17 \mathrm{~cm}$ high, $18 \mathrm{~cm}$ wide at the top and $4.5 \mathrm{~cm}$ wide at the bottom) divided by a sliding door into a lit compartment $(35 \mathrm{~cm})$ and a dark compartment $(60 \mathrm{~cm})$ as described elsewhere (Liang, 1999). A cage made of 1-cmthick transparent blue acrylic $(27 \mathrm{~cm}$ long, $22 \mathrm{~cm}$ wide and $35 \mathrm{~cm}$ high) was placed alongside the apparatus to serve as the waiting place. After surgery, rats were individually placed into this waiting cage without being connected to the unit recording cable for $1 \mathrm{~h} /$ day for 4 consecutive days in order to become adapted to the waiting environment and to being handled.

To facilitate unit recording during performance of the task, a modified training and testing procedure for the one-trial step-through inhibitory avoidance task was adopted. This modification allowed simultaneous unit recording and behavioural assessment in a freely moving animal as well as collection of sufficient data on neuronal firing as the rat was situated in different conditions. Recording sessions formally started 7-10 days after surgery as the rat had been adapted to the waiting environment. From Day 1 to Day 3, the rat was placed into the waiting cage with the recording cable plugged in. Recording started at least $10 \mathrm{~min}$ after entering, at which time no apparent walking was observed. After $5 \mathrm{~min}$ of unit recording in the waiting cage, the rat with the cable connected was placed into the lit compartment of the task apparatus, facing away from the sliding door. 


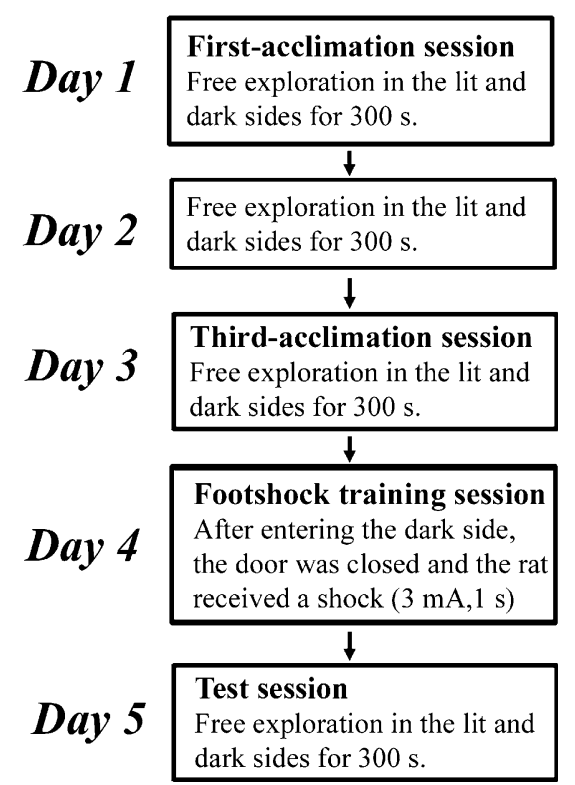

FIG. 2. A schematic diagram for the experimental protocol. The control group followed the same procedure except that the footshock training session was performed in an environment other than the task apparatus for acclimation and testing.

As the rat turned around, the door was opened and the rat was allowed 5 min to freely explore both sides of the alley. At the termination of each acclimation session, the rat was put back into the waiting cage for a further $5 \mathrm{~min}$ of unit recording.

The cable was then unplugged and the rat was returned to its home cage. The first 5-min period of unit recording in the waiting cage before entering the task apparatus was denoted the 'pre-task period', and the $5 \mathrm{~min}$ of free exploration in the task apparatus was denoted the 'task period'. The 5-min period of unit recording in the waiting cage after leaving the task apparatus was denoted the 'post-task period'. The first and the third times for a rat to be subjected to the task procedure (on Day 1 and Day 3, respectively) were denoted, respectively, the first and the third acclimation sessions. Unit activities were recorded in these two sessions to assess possible effects of habituation to the apparatus. On Day 4, a footshock training session was carried out with the same procedure as that in the acclimation sessions except that, as the rat entered the dark side, the door was closed and a 3-mA shock (a $60-\mathrm{Hz}$ square wave administered by a Grass S48 stimulator; Astro-Medical, Inc. West Warick, RI, USA) was delivered through the floor plate for $1 \mathrm{~s}$ to each rat of the experimental group $(n=9)$. The rat was constrained in the dark side for another $30 \mathrm{~s}$ and then returned to the waiting cage. On Day 5, a test session was carried out following exactly the same procedure of the acclimation session. The initial stepthrough latencies and the total time spent in the lit side during the 5-min task period were measured to assess the behavioural effects of learning in the first acclimation, third acclimation and test sessions for a given rat.

The control group $(n=7)$ went through the same procedure except that on the training day they received the shock in another box (18.5 cm long, $10 \mathrm{~cm}$ wide and $30 \mathrm{~cm}$ high), which was situated in a large laboratory room under ordinary indoor illumination. This setting was to maximize distinctions in the contextual cues associated with the shock between the control and experimental conditions and thus to avoid any generalization of the aversive experience for the control group. Figure 2 diagrammatically summarizes the experimental protocol, in which the experimental and control groups had essentially equivalent experience except that for the experimental group the footshock was contingent upon the step-through behaviour and changes of the light-dark cues in the task apparatus.

\section{Histology}

At the conclusion of each experiment, the rat was deeply anaesthetized with an overdose of sodium pentobarbital. The best-recorded channel on each side was marked with an electrolytic lesion by passing an anodal current of $100 \mu \mathrm{A}$ for $15 \mathrm{~s}$. Then the rat was perfused through the heart with physiological saline followed by $10 \%$ formalin. The brain was removed and stored in $10 \%$ formalin with $30 \%$ sucrose for at least $24 \mathrm{~h}$. Frozen sections of $100 \mu \mathrm{m}$ were taken and slices from the target region were stained using the acetylcholinesterase method (Woof \& Butcher, 1981). Recording sites were reconstructed with camera lucida based on lesions generated by the marked wire.

\section{Data analysis}

Behaviour

Differences between the control and experimental groups in the task behaviour for the first acclimation, third acclimation and test sessions were assessed from two measurements: the initial step-through latency and total time spent in the lit side. The central tendency and dispersion of such data were represented, respectively, by the median value and interquartile range, while differences were analysed with nonparametric statistics.

\section{Unit activity}

In order to trace the activity change of a single unit throughout the 5 recording days, cluster boundaries of isolated units computed from the third acclimation session were applied to the first acclimation session and the test session (Maren, 2000). Ninety-five single units from the experimental group and 49 single units from the control group could be traced over the 5-day recording period. Data for unit activity are presented as the mean \pm SEM. Changes in mean firing rates of the recorded units in the control and experimental groups were analysed using three-way ANOVAs with the group as the betweensubject variable and period and session as the within-subject variables. Post hoc comparisons were performed by using the StudentNewman-Keuls method whenever an overall significant effect was detected.

\section{Results}

\section{Distribution of recording sites}

Recording bundles were implanted into the amygdala on both sides in seven control rats and seven experimental rats. In two other experimental rats, electrodes were implanted only into the right amygdala. Data from two bundles (one aimed at the left amygdala of an experimental rat and the other aimed at the left amygdala of a control rat) were discarded due to misplacement.

A typical example of a lesion at the electrode tip is shown in Fig. 3A. Every lesion site had been recovered. The distributions of the reconstructed lesion sites (large dots or circles) and the identifiable tips of the recording tracks (small dots or circles) for the control (open circles) and experimental (solid dots) groups are shown in Fig. 3B and $\mathrm{C}$, respectively. No apparent difference in distribution between the groups was noticed. 

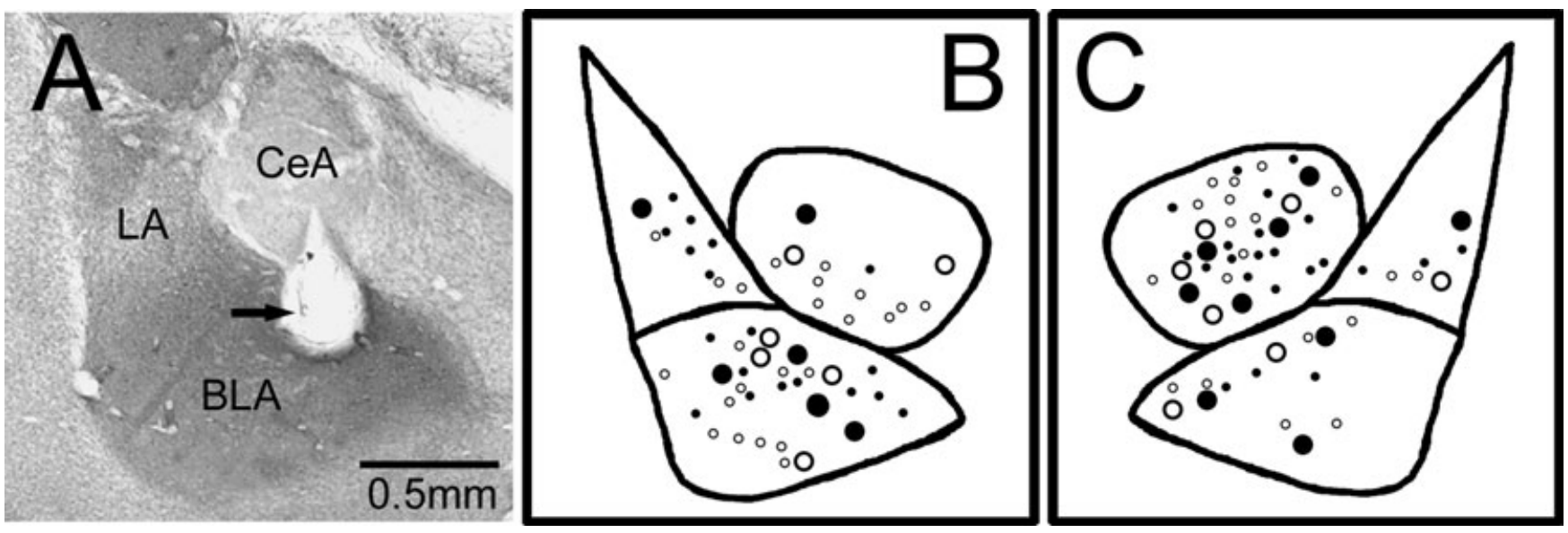

FIG. 3. Histological verification of the recording sites. (A) The left panel shows a photomicrograph of a typical lesion site (arrow) in the left basolateral nucleus of the amygdala stained by the acetylcholinesterase histochemical method. (B and C) The distribution of the lesion sites are indicated by large dots and the identifiable tips of recording tracks are indicated by small dots. The locations in the left (B) and the right (C) amygdala are plotted for the experimental group (filled symbols) and the control group (open symbols), respectively.

\section{Behaviour changes in the inhibitory avoidance task}

Rats readily acquired the inhibitory avoidance response after a single foot shock administered in the dark side. The performance indicated by the initial step-through latency is shown in Fig. 4A. The Friedman's rank test detected significant differences in the initial step-through latencies among the various task sessions in the experimental group $\left(\chi_{2}^{2}=6.23, P<0.05\right)$, but not in the control group $\left(\chi_{2}^{2}=3.70\right.$, $P>0.05$ ). Furthermore, Newman-Keuls tests indicated that for the experimental group, the initial step-through latencies were significantly longer in the test session than those in either the first or third acclimation session $(P<0.05)$, while the latter two did not differ from each other. The Wilcoxon rank-sum test indicated that rats in the experimental group stayed for a significantly longer period of time in the lit site before their first entry into the dark side than did the controls $(t=35.0, P<0.05)$ during the test session, but no such difference was found in the first or third acclimation session.

The total time spent in the lit compartment at various sessions is shown in Fig. 4B. The Friedman's rank test revealed significant differences among the various task sessions for the experimental group $\left(\chi_{2}^{2}=13.6, P<0.001\right)$ but not for the control group $\left(\chi_{2}^{2}=4.57\right.$, $P>0.05)$. Furthermore, Newman-Keuls tests indicated that the time spent in the lit compartment for the experimental group was significantly longer during the test session than those during either the first or third acclimation session $(P<0.05)$, while the latter two did not differ from each other. The Wilcoxon rank-sum test indicated that, during the test session, rats in the experimental group spent a significantly longer period of time in the lit side than did the controls $(t=28.0, P=0.001)$.

On the basis of the above analyses, both measures of avoidance behaviour yielded consistent results indicating that the experimental group, but not the control group, showed retention of the aversive experience received in the task apparatus during the training session.

\section{Changes in neuronal activities in the inhibitory avoidance task}

To categorize units recorded from the amygdala into principal neurons and interneurons, a criterion proposed by previous studies on cats was adopted, namely, cells with high spontaneous firing rates $(>5 \mathrm{~Hz})$ were classified as interneurons (McDonald, 1992; Paré \& Gaudreau,
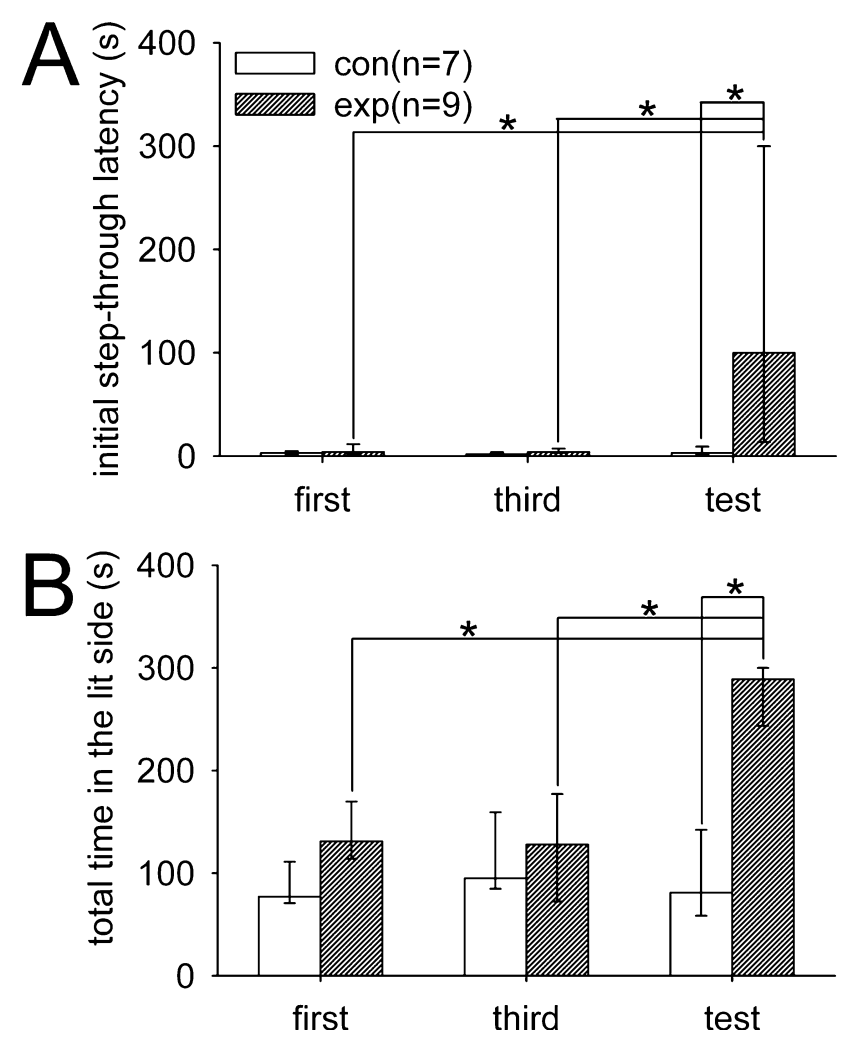

FIG. 4. Effect of footshock training on (A) initial step-through latencies into the dark side and (B) the total time spent in the lit side of the task apparatus in the retention test. Performance is expressed by the median and the interquartile range of each group. ${ }^{*} P<0.05$.

1996; Collins \& Paré, 1999). Of the units recorded in these studies, the majority $(>80 \%)$ were principal neurons characterized by low spontaneous firing rates (usually $<1 \mathrm{~Hz}$ ), whereas interneurons characterized by tonic, high spontaneous firing rates (mostly $>10 \mathrm{~Hz}$ ) were the minority.

In total, 144 units were traced throughout the 5-day recording period in the present study. Among them, 95 units were in the 


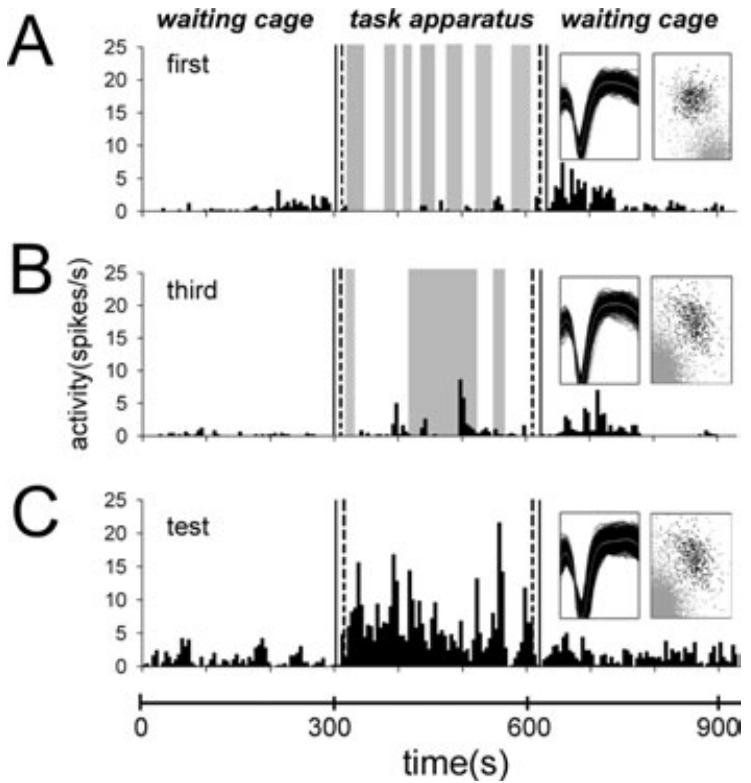

FIG. 5. Rate histograms of a representative amygdaloid unit in the left basolateral nucleus throughout the three sessions in the inhibitory avoidance task for an experimental rat. From top to bottom are the rate histograms in (A) the first acclimation session, (B) the third acclimation session and (C) the test session. Bin size, $5 \mathrm{~s}$. Solid vertical lines denote the time that the rat was removed from or placed into the waiting cage. Dashed vertical lines denote the start and the end of the task periods. The cumulative waveforms and the principal component plots of the unit in each task session are shown as insets in the right panel. Behaviourally the rat remained in the lit side for the entire test session, in sharp contrast to its previous behaviour of moving back and forth between the lit side and the dark side (shaded areas). Note the marked increased unit activity in the task period during the test session.

experimental group and 49 were units in the control group. Consistent with previous findings, only a small proportion of the recorded neurons ( $n=10,6.9 \%$ of our sample) were interneurons. Of the 10 interneurons, three (one in the right CeA and two in the left BLC) were in the control group and seven (four in the right $\mathrm{CeA}$ and three in the left BLC) were in the experimental group. Data from the two neuron types were pooled for further analyses without treating interneurons separately due to the small sample size and lack of apparent changes in patterns distinctive from the other neurons across the recording period.

Figure 5 shows one example of a unit recorded from the left BLC; the activity of this unit profoundly increased during the task period in the test session.

Figure 6 shows the results of the average activities of all single units during different task periods of different task sessions for the control and experimental groups. Data were analysed using three-way ANOVAs with group (control and experimental) as the between-subjects variable, and period (pre-task, task and post-task) and session (first acclimation, third acclimation and test) as the within-subject variables.

In the control group, the results indicated that firing rates in amygdala neurons increased as the rat entered the task apparatus initially, but this augmentation gradually subsided with repeated entries into the task environment; hence the neurons showed habituation to novelty in their activity. Likewise, the rise in amygdala activity upon entering the task apparatus also showed habituation during acclimation phase in the experimental group. However, in contrast to what was found in the controls, average activities in the amygdala rose again when the experimental rats entered the apparatus after having received footshock training in it.
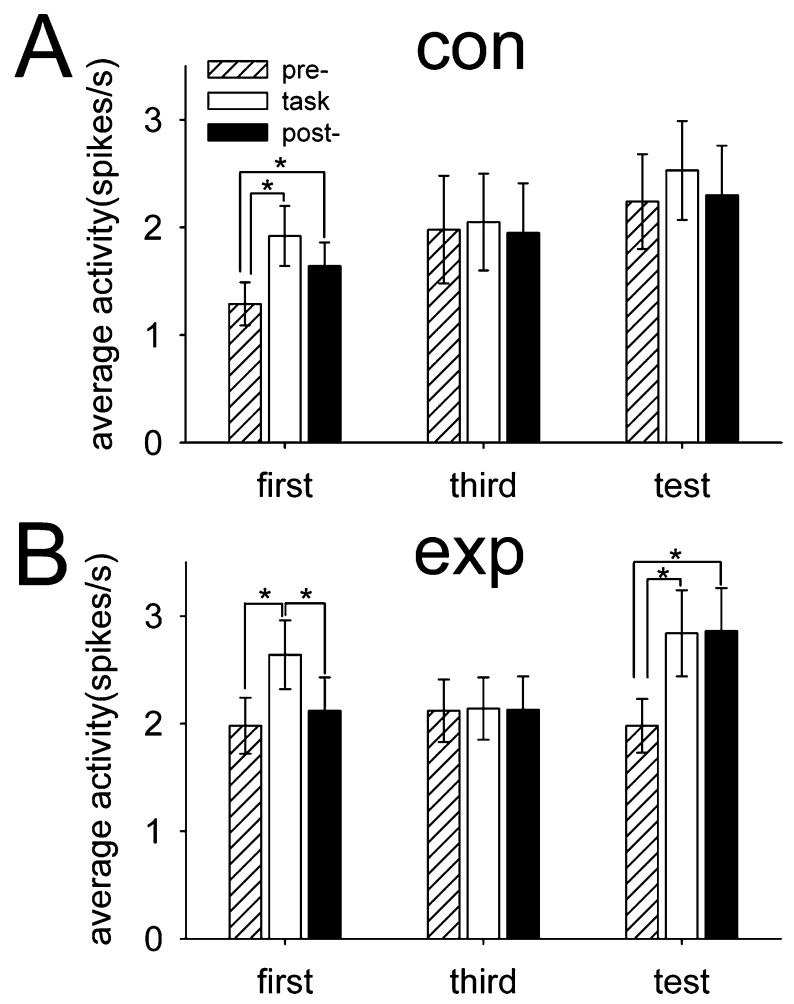

FIG. 6. Average activities of single units during different task periods and task sessions in (A) the control and (B) experimental groups. Bars are mean \pm$\mathrm{SEM} ; * P<0.05$.

Statistical analysis revealed significance for the main effect of period $\left(F_{2,284}=9.11, P<0.0002\right)$ and the two-way interaction effect of period and session $\left(F_{4,568}=5.6, P=0.0002\right)$. Furthermore, the three-way interaction was also significant $\left(F_{4,568}=3.3, P<0.02\right)$, suggesting that the experimental and control groups showed different patterns of activity changes across the various periods over the sessions. Post hoc paired comparisons revealed that, in the first acclimation session, the control group had significantly higher average activities during the task or post-task periods than during the pre-task period, while the experimental group had significantly higher average activities during the task period than during the pre-task or post-task periods (all $P$-values $<0.05$ ). In the third acclimation session, no significant increase in average activities over the pre-task baseline was detected in the task or post-task period for either group (all $P$-values $>0.05)$. The most critical difference occurred in the test session: in the control group, average activities showed no significant differences among the three periods $(P>0.05)$; in contrast, in the experimental group the average activities during the task or post-task period were significantly higher than those during the pre-task period $(P<0.05)$.

Among the nine rats in the experimental group, four of them never stepped into the dark side of the training apparatus during the test session and were categorized as the no-entry subgroup, while the other five which did were categorized as the entry subgroup. Of the 56 units recorded from the entry subgroup, the averaged activity in the lit side was significantly higher than that in the dark side according to paired $t$-test $\left(t_{55}=2.46, P<0.05\right)$. With further analyses of single-unit activities of the entry subgroup it was observed that, although the firing rate was more pronounced in the lit side than in the dark side as a whole, few units actually showed apparent increases in firing rates during the test session. Of the 56 units recorded in the entry subgroup, 10 units (three in the right $\mathrm{CeA}$, three in the left $\mathrm{CeA}$, two in the right 


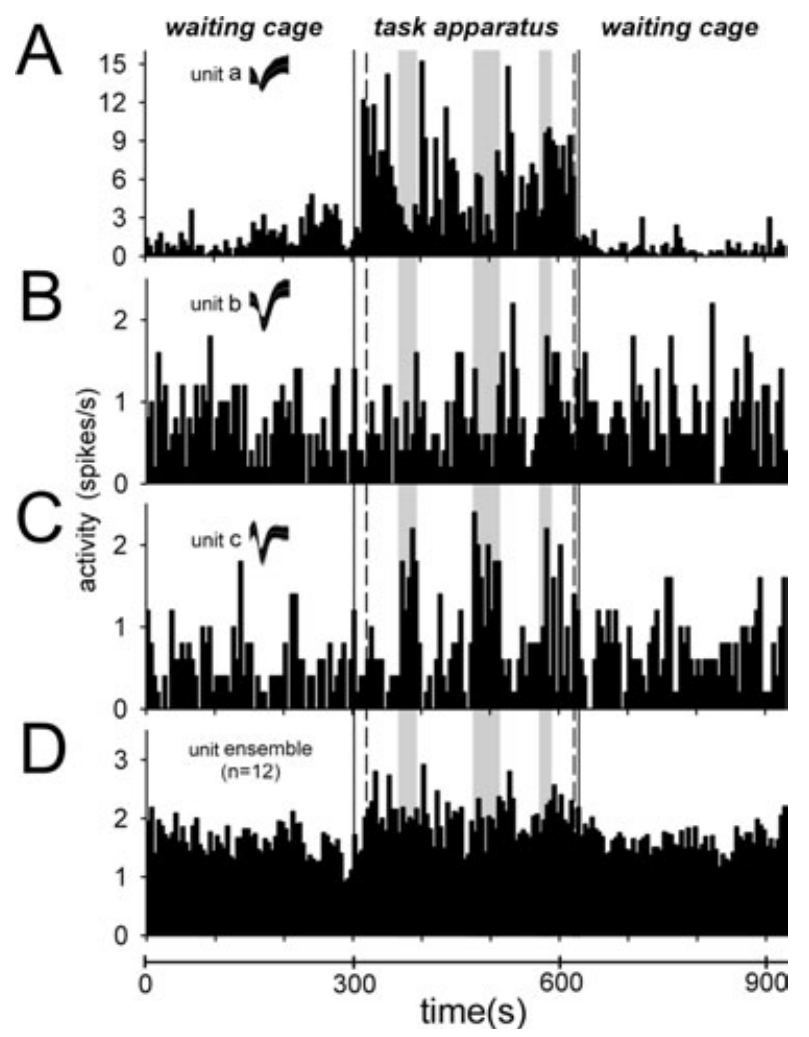

FIG. 7. Single-unit and ensemble activities recorded from a rat in the experimental group during the test session. (A) Unit (a) fired more prominently in the lit side, (B) unit (b) did not show firing preference in either the lit or dark side, and (C) unit (c) fired more prominently in the dark side of the task apparatus. (D) Average ensemble activities of 12 amygdala units recorded simultaneously in the same rat. Note the increase in the average ensemble amygdala activity in the task apparatus. Refer to legend of Fig. 5 for other descriptions.

BLC and two in the left BLC) showed a general increase in the task apparatus regardless of the rat's position in the lit or dark side (with a firing pattern similar to the unit shown in Fig. 5 at the test session), two units (one in the right $\mathrm{CeA}$ and one in the left $\mathrm{BLC}$ ) showed firing preference for the dark side, and only one unit (in the left BLC) showed a firing preference for the lit side.

Figure 7 shows the ensemble unit activities recorded in one animal in the entry subgroup and the three single units isolated from such activity in this rat. Unit (a) (Fig. 7A) fired more profoundly in the lit side and unit (c) (Fig. 7C) fired more profoundly in the dark side, while unit (b) showed no obvious preference in firing for the lit or dark side (Fig. 7B). Mean ensemble activities of 12 units (Fig. 7D) showed an overall increase in firing in the task apparatus compared to that in the waiting cage for this rat. These results suggest that, while individual units may show differential firing preferences in either the lit or dark side, there was a trend for overall firing to increase in the task apparatus.

To further characterize the effect of experience on unit activity, units in Fig. 7 were traced back to the first and third acclimation sessions. Figure 8 depicts the behaviour of unit (a) across the sessions. This unit showed preference for the lit side when the rat first entered the task apparatus, but the preference had subsided by the third acclimation session and then recurred during the test session after shock training. Figure 9 depicts the behaviour of unit (c) across the sessions. This unit showed no preference during the acclimation sessions, but a dark side preference appeared after the shock training. Another unit showing a

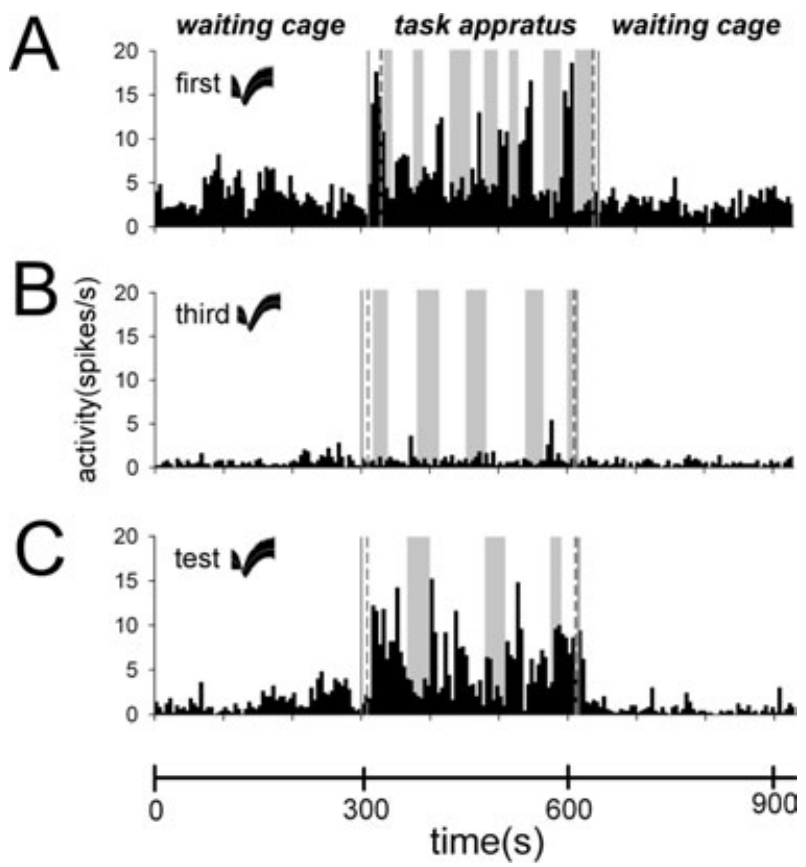

FIG. 8. Rate histograms of unit (a) in Fig. 7 throughout the three sessions in the inhibitory avoidance task. Refer to legend of Fig. 5 for other descriptions. Note that this unit showed a light preference early in the first acclimation session, but this subsided in the third acclimation session. This preference reappeared in the test session.

dark preference during the test session showed no preference during the acclimation sessions (data not shown).

Because the five rats in the entry subgroup shuttled several times between the lit and the dark sides of the task apparatus during the test session, as did the seven control rats, we pursued further whether there were consistent changes in the transient ensemble firing rate over the

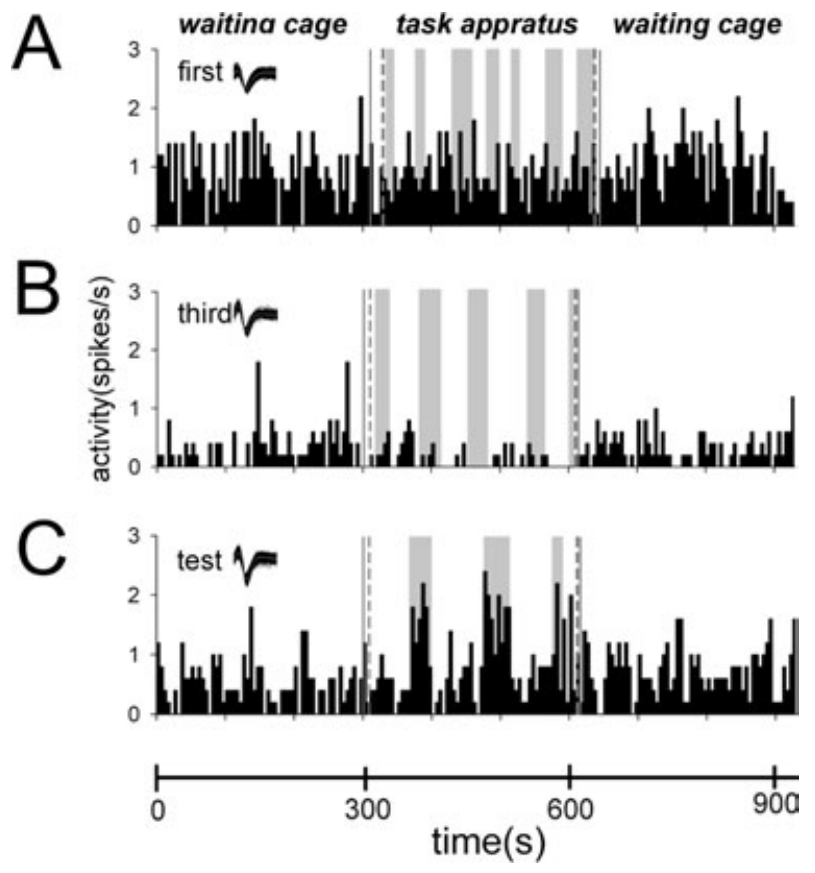

FIG. 9. Rate histograms of unit (c) in Fig. 7 throughout the three sessions in the inhibitory avoidance task. Refer to legend of Fig. 5 for other description. Note that the dark preference did not occur in the acclimation sessions. 
TABLE 1. Unit activity as the rat crossed from the lit side to the dark side and vice versa during the test session

\begin{tabular}{llll}
\hline & \multicolumn{2}{l}{ Change in unit activity (firing rate) } \\
\cline { 2 - 4 } $\begin{array}{l}\text { Group and direction } \\
\text { of movement }\end{array}$ & Increased & No change & Decreased \\
\hline $\begin{array}{l}\text { Control } \\
\text { Lit to dark }\end{array}$ & 14 & 1 & \\
$\quad$ Dark to lit & 16 & 1 & 18 \\
Experimental & & & 15 \\
$\quad$ Lit to dark & 5 & 2 & 9 \\
Dark to lit & 11 & 0 & 5 \\
\hline
\end{tabular}

various occasions as the rat stepped into or withdrew from the dark side. For rats in the control group and the entry subgroup, we compared the 5-s ensemble firing rates in the amygdala of each rat immediately before and after leaving the lit side to enter the dark side or immediately before and after returning from the dark side to the lit side. The seven rats in the control group in total entered the dark side 33 times and moved back 32 times. The five rats in the entry subgroup entered the dark side 16 times and moved back 16 times. The average shuttle frequency was higher in the control group (4.64 times per rat) than in the entry subgroup (3.2 times per rat). Increases, no-changes and decreases in firing rates were observed in both the control group and the entry subgroup. The frequency distribution of the three types of firing rate changes as rats shuttled between the two sides are shown in Table 1. The difference in distribution was not significant for the control groups $\left(\chi_{2}^{2}=0.391, P>0.05\right)$ but approached statistical significance $\left(\chi_{2}^{2}=5.393, P=0.067\right)$ for the entry subgroup. This result implied that as rats in the entry subgroup were leaving the lit side for the dark side ensemble firing rates in the amygdala showed a tendency to decrease, while as rats were returning from the dark side back to the lit side, ensemble firing rates showed a tendency to increase, consistent with the finding of higher firing rate in the lit side.

Finally, average activities were analysed by individual two-way ANOVAs to assess the effects of several between-subjects variables such as subgroup (in the lit box: no-entry, $n=39$; entry, $n=56$ ), subnuclei (CeA, $n=42$; BLC, $n=53$ ), or hemisphere (left, $n=53$; right, $n=42$ ) across the three periods of the test session. All analyses consistently detected a significant main effect of periods $(P<0.001)$, yet detected no significant main effect of subgroup, subnuclei or hemisphere, nor significant interaction between any of these variables and periods $(F<1)$. Therefore, the entry and no-entry subgroups, the $\mathrm{CeA}$ and $\mathrm{BLC}$, and the left and right amygdala had similar firing rates.

\section{Discussion}

This study was aimed at investigating the amygdaloid single-unit activity in the step-through inhibitory avoidance task. Our results indicate that, as the animal encountered the novel task apparatus for the first time, activity increased in some amygdala neurons. As the rat adapted to the task environment after two sessions of acclimation, the overall firing rate in these neurons returned to the baseline. Footshock training in the task apparatus rekindled the increase in the firing rate bilaterally in both the CeA and BLC. Thus the present findings suggest that habituation and inhibitory avoidance learning modified neuronal activity in the amygdala.

In contrast to the conventional inhibitory avoidance procedure that terminates a trial once the rat steps into the dark side, the present study allowed the rat to move freely in the task apparatus for $300 \mathrm{~s}$ and recorded the total time spent in the lit side as retention indices in addition to the typical initial step-through latency. This modification provided a longer sampling time for unit activity recording and hence avoided any spurious changes in unit activity that might have occurred in a shorter sampling period. The present results showed that both retention indices yielded congruent findings, suggesting that the modified paradigm is essentially compatible with the conventional one.

Several previous studies have reported engagement of amygdaloid neurons in classical fear conditioning (Quirk et al., 1995, 1997; Repa et al., 2001; Goosens et al., 2003); however, similar findings on the inhibitory avoidance task are not available in the literature. Such studies might be impeded by factors including the lack of a discrete and distinctive cue (i.e. the conditioned stimulus) to temporally register the evoked responses of units with ease, the single learning trial prohibiting detection of correlative growth between neural and behavioural changes across trials, and the difficulties of recording unit activity in a freely moving animal under a shock condition. This study, to the best of our knowledge, constitutes the first report on the single-unit activities of amygdala neurons in rats before and after inhibitory avoidance learning.

Our data indicated that the amygdala neurons showed an increased firing rate when the rat was exposed to the task apparatus for the first time. For some rats, the increased firing even persisted after the rat was returned to the well-adapted waiting cage, consistent with previous findings that novel stimuli have a sustained impact on amygdala neurons (O'Keefe \& Bouma, 1969). Yet this marked response to novelty readily habituated. At the third acclimation session, amygdala neurons showed no increased firing during the task or post-task period (Fig. 4), even though the initial step-through latency or total time spent in the lit side remained largely the same over these sessions. These data attest to the necessity of preadaptation to the task environment in achieving a stable baseline of neuronal activity in rats against which the learning effect can be detected.

Some previous findings implicate the amygdala in habituation learning (Tamásy et al., 1975). For those amygdala neurons responding to novel stimuli, some readily showed habituation while others did not (Ben-Ari \& Le Gal La Salle, 1974; Rosenkranz \& Grace, 2002). However, perturbing the amygdala has no effect on acquiring or expressing the habituated responses to novelty (Da Cunha et al., 1991; Wolfman et al., 1991; Rosat et al., 1992). Such findings raise the issue of whether the observed activity change was locally initiated in the amygdala or downstream to the plasticity of a remote site implicated in habituation, such as the hippocampus (Da Cunha et al., 1991; Rosat et al., 1992). Thus, a causal role of the amygdala in habituation to novelty remains to be further tested.

In contrast to the reduction of elevated firing by the acclimation procedure, our across-day analysis of ensemble activity showed that, after footshock training, the firing rate significantly increased over the pre-task baseline in the retention test (Figs 6B and 7D). Such an increase was specific to the training experience because no corresponding changes were detected in the control group receiving the shock in a different context. The increased activity did not subside when the rat returned to the waiting cage at the post-task period on the testing day (Fig. 6B), attesting once more to the persistence of the evoked activity in the amygdala neurons. Previous findings reported that within a single training session of classical conditioning some amygdala neurons altered their activity only in the early phase of training while others showed changes persisting until the late phase of a training session or even after over-training (Repa et al., 2001). The present findings extend our knowledge by showing that certain plastic changes in amygdala neurons might endure at least 1 day after a single training trial of an inhibitory avoidance task.

Our analysis showed that, for the entry subgroup, firing in the lit side was more pronounced than that in the dark when the 56 units 
recorded from these animals were treated as a whole. As noted in the results, when the behaviour-contingent firing pattern was analysed in an animal effectively recalling the shock experience, amygdala units showed three patterns of activation: more excited in the lit side (Fig. 7A), more excited in the dark side (Fig. 7C) or equally excited in both sides, with the last being the most frequent. On the basis of single-unit activities, 13 out of 56 units in the entry subgroup showed dramatic firing changes during the test session. In the no-entry subgroup, four out of 39 units showed such dramatic firing changes in the lit side during the test session (two in the right BLC and two in the left BLC). Thus, on average, $18 \%$ of the sampled neurons (17 of 95) could be counted as highly 'responsive' for retention of an inhibitory avoidance task. These 17 units had an increase in firing rate of $4.84 \pm 0.98 \mathrm{~Hz}$ (mean $\pm \mathrm{SEM}$ ) over their pre-task baseline. For the 49 units recorded from the control group, only one of them showing activity change fell into the $95 \%$ confidence interval of this 'responsive' unit distribution.

Previous studies indicated that, in the basolateral amygdala, $26 \%$ of the sampled neurons were responsive to acquisition of olfactory discrimination (Schoenbaum et al., 1999) and 43\% were responsive to acquisition of cocaine self-administration (Carelli et al., 2003). These findings are consistent with the notion that the amygdala is innervated by multiple sensory inputs (Pitkänen, 2000) and is involved in multiple types of learning tasks. These data, if considered across studies, raise the possibility that various types of learning may differentially activate amygdala neurons. It would be of great interest in the future to study whether different learning demands engage distinctive populations of neurons in the amygdala or the same population of neurons with different efficiencies. Experiments are presently under way to address this question.

That some amygdala neurons responded differentially to the lit and dark sectors of the task apparatus raises two interesting questions: are such patterns intrinsic or forged by experience and do they bear any relationship to the test performance? As indicated in Figs 8 and 9, in one neuron differential firing in the two sectors was present at the beginning, subsided during acclimation but reappeared after training. In another neuron, the differential firing in the two sectors appeared only after training. Thus, the unique firing patterns detected in amygdala neurons of a trained animal, whether existing before training or not, are nonetheless subjected to modification with experience.

Concerning the second question, results from Table 1 reveal a trend in the experimental group that the firing rate in an ensemble of neurons tended to decrease as the rat moved from the lit into the dark side and to increase as the rat moved in the reverse direction. No such changes were detected in the control group. While such results appear to suggest a weak relationship between the lit side and the more vigorous transient activity, it remains to be determined whether such an activity change is the antecedent or the consequence of the rat changing its location.

The present results detected altered neuronal activity in both the $\mathrm{CeA}$ and BLC. Evidence has suggested that different amygdala circuits are involved in classical and operant conditioning tasks. The basolateral amygdala nuclei are part of a system subserving voluntary or intentional choice responses to emotional events based on operant conditioning, while the $\mathrm{CeA}$ is more involved in reflexive, automatic responses evoked by salient stimuli acquired via classical conditioning (Killcross et al., 1997). Previous findings showed that retention in the inhibitory avoidance task was more susceptible to post-training treatments applied to the basolateral nuclei of the amygdala but impervious to those applied to the CeA (McGaugh et al., 2000). These data imply a predominance of an operant component in the inhibitory avoidance task. However, evidence has demonstrated that classical conditioning also contributed significantly to inhibitory avoidance memory (Liang, 1999). Further studies from this laboratory have shown that pretraining lesions of either the BLC or CeA compromise the retention of this response (Chen et al., 2002). Our results for the unit activity suggest that both types of learning may be recruited in acquiring an inhibitory avoidance response; this is predicted by the two-process theory of avoidance learning (Levis, 1989).

Lateralization of brain function in transacting affective events has been demonstrated in humans and animals (Zald, 2003). Previous findings have shown that in albino rats, acquisition, consolidation or expression of memory of an aversive experience was more vulnerable to suppression of the right than the left amygdala by local infusion of lidocaine or muscimol (Coleman-Mesches \& McGaugh, 1995a,b,c; Coleman-Mesches et al., 1996). If such data were viewed as the right amygdala having a more critical role in coding the learned information then the learning-induced activity change should have been more prominent in the right amygdala. However, we failed to find differential responsiveness of the left or right CeA or BLC during the test session in the experimental group. A possible interpretation is that these areas are interconnected such that increased firing in one side would lead to an increase in the other. Alternatively, manipulation applied to the right amygdala may exert its influence on memory by altering neural activity subserving memory storage processes elsewhere in the brain rather than the in situ activity.

A critical issue that remains to be addressed is whether the observed neuronal changes per se are responsible for recalling the learned information and expressing correct behaviour in a retention test. Previous evidence has shown that inactivation of the amygdala with lidocaine or an AMPA ( $\alpha$-amino-3-hydroxy-5-methyl-4 isoxazole propionic acid) antagonist, CNQX (6-cyano-7-nitroquinoxaline), at the time of a 1-day retention test induced memory expression deficits (Liang et al., 1996; Izquierdo et al., 1997), implying that successful operation of avoidance memory, at least 1 day after its acquisition, requires integrated functioning of certain amygdala neurons. Thus, the activity changes detected in the amygdala might be viewed as the neural trace of learning, and lidocaine or CNQX infused into the amygdala disrupts the overall excitation or intricate activity pattern required for successful retrieval. Alternatively, in view of similar responses in the amygdala observed for the first acclimation and the test session, the augmented activity recorded may represent fear or stress responses per se, and lidocaine or CNQX may simply block the final common output of fear.

In summary, the present study, by recording multiple single-unit activities in the amygdala of freely moving rats, clearly demonstrated activity changes during habituation and inhibitory avoidance learning. Some studies have argued that activity changes in the amygdala alone or in cooperation with its connected sites such as the nucleus accumbens may subserve the learned experience and bear a causal link with the avoidance behaviour (Schoenbaum et al., 1999; Setlow et al., 2003). However, before all issues raised above are satisfactorily resolved, such a view remains at best only one of the many tenable interpretations afforded by correlative data, as recently argued by Paré (2002) for the case of Pavlovian fear conditioning.

\section{Acknowledgements}

This study was supported by grants of Center Project from the Cognitive Science of the Academia Sinica of the R.O.C. (to C.T.Y. and K.C.L.), and NSC91-2311-B-002-051 (to C.T.Y.), NSC-91-2413-H-002-020 and NSC-932752-H-002-006-PAE (to K.C.L.) from the National Science Council of the R.O.C. The authors would like to thank Dr Deh-Yu Chen for his valuable advice on statistics, as well as Mr Cheng-Chiu Huang and Ms Chun-Chun Chen for their assistance in development of the modified behavioural paradigm in the inhibitory avoidance task. 


\section{Abbreviations}

$\mathrm{CeA}$, central nucleus of the amygdala; BLC, basolateral complex of the amygdala.

\section{References}

Aggleton, J.P. (2000) The Amygdala: a Functional Analysis, 2nd edn. Oxford University Press, New York.

Ben-Ari, Y. \& Le Gal La Salle, G. (1974) Lateral amygdala unit activity. II. Habituating and non-habituation neurons. Electroenceph. Clin. Neurophysiol., 37, 463-472.

Cahill, L. \& McGaugh, J.L. (1998) Mechanisms of emotional arousal and lasting declarative memory. Trends Neurosci., 21, 294-299.

Carelli, R.M., Williams, J.G. \& Hollander, J.A. (2003) Basolateral amygdala neurons encode cocaine self-administration and cocaine-associated cues. J. Neurosci., 23, 8204-8211.

Chen, C.C., Chang, C.H., Yen, C.T. \& Liang, K.C. (2002) Involvement of amygdala nuclei in learning inhibitory avoidance tasks. Soc. Neurosci. Abstr. 284, 19.

Coleman-Mesches, K. \& McGaugh, J.L. (1995a) Differential involvement of the right and left amygdala in expression of memory for aversively motivated training. Brain Res., 670, 75-81.

Coleman-Mesches, K. \& McGaugh, J.L. (1995b) Muscimol injected into the right or left amygdaloid complex differentially affects retention performance following aversively motivated training. Brain Res., 676, 183-188.

Coleman-Mesches, K. \& McGaugh, J.L. (1995c) Differential effects of pretraining inactivation of the right or left amygdala on retention of inhibitory avoidance training. Behav. Neurosci., 109, 642-647.

Coleman-Mesches, K., Salinas, J.A. \& McGaugh, J.L. (1996) Unilateral amygdala inactivation after training attenuates memory for reduced reward. Behav. Brain Res., 77, 175-180.

Collins, D.R. \& Paré, D. (1999) Reciprocal changes in the firing probability of lateral and central medial amygdala neurons. J. Neurosci., 19, 836-844.

Da Cunha, C., Wolfman, C., Huang, C.H., Walz, R., Koya, R., Bianchin, M., Medina, J.H. \& Izquierdo, I. (1991) Effect of post-training injections of flumazenil into the amygdala, hippocampus and septum on retention of habituation and of inhibitory avoidance in rats. Braz. J. Med. Biol. Res., 24, 301-306.

Fanselow, M.S. \& LeDoux, J.E. (1999) Why we think plasticity underlying Pavlovian fear conditioning occurs in the basolateral amygdala. Neuron, 23, 229-232.

Goosens, K.A., Hobin, J.A. \& Maren, S. (2003) Auditory-evoked spike firing in the lateral amygdala and Pavlovian fear conditioning: mnemonic code or fear bias? Neuron, 40, 1013-1022.

Izquierdo, I., Quillfeldt, J.A., Zanatta, M.S., Quevedo, J., Schaeffer, E., Schmitz, P.K. \& Medina, J.H. (1997) Sequential role of hippocampus and amygdala, entorhinal cortex and parietal cortex in formation and retrieval of memory for inhibitory avoidance in rats. Eur. J. Neurosci., 9, 786-793.

Killcross, S., Robbins, T.W. \& Everitt, B.J. (1997) Different types of fearconditioned behaviour mediated by separate nuclei within amygdala. Nature, 388, 377-380.

LeDoux, J.E. (2000) Emotion circuits in the brain. Annu. Rev. Neurosci., 23, $155-184$

Levis, D.J. (1989) The case for a return to a two-factor theory of avoidance: The failure of non-fear interpretations. In Lein, S.B. \& Mowerer, R.R. (eds), Contemporary Learning Theories: Pavlovian Conditioning and the Status of Traditional Learning Theory. Lawrence Erlbaum Associates, New Jersey, pp. 227-277.

Liang, K.C. (1999) Pre- or post-training injection of buspirone impaired retention in the inhibitory avoidance task: involvement of amygdala $5-\mathrm{HT}_{1 \mathrm{~A}}$ receptors. Eur. J. Neurosci., 11, 1491-1500.

Liang, K.C., Hon, W. \& Davis, M. (1994) Pre- and posttraining infusion of $\mathrm{N}$-methyl-D-aspartate receptor antagonists into the amygdala impair memory in an inhibitory avoidance task. Behav. Neurosci., 108, 241-253.

Liang, K.C., Hu, S.J. \& Chang, S.C. (1996) Formation and retrieval of inhibitory avoidance memory: differential roles of glutamate receptors in the amygdala and medial prefrontal cortex. Chinese J. Physiol., 39, 155-166.

Liang, K.C., Juler, R.G. \& McGaugh, J.L. (1986) Modulating effects of posttraining epinephrine on memory: involvement of the amygdala noradrenergic system. Brain Res., 368, 125-133.

Liang, K.C., McGaugh, J.L. \& Yao, H. (1990) Involvement of amygdala pathways in the influence of posttraining amygdala norepinephrine and peripheral epinephrine on memory storage. Brain Res., 508, 225-233.
Maren, S. (2000) Auditory fear conditioning increases CS-elicited spike firing in lateral amygdala neurons even after extensive overtraining. Eur. J. Neurosci., 12, 4047-4054.

Maren, S. (2001) Neurobiology of Pavlovian fear conditioning. Annu. Rev. Neurosci., 24, 897-931.

Maren, S., Poremba, A. \& Gabriel, M. (1991) Basolateral amygdaloid multiunit neuronal correlates of discriminative avoidance learning in rabbits. Brain Res., 549, 311-316.

McDonald, A.J. (1992) Cell types and intrinsic connections of the amygdala. In Aggleton, J.P. (ed.), The Amygdala: Neurobiological Aspects of Emotion, Memory, and Mental Dysfunction. Wiley-Liss, New York, pp. 67-96.

McGaugh, J.L. (2004) The amygdala modulates the consolidation of memories of emotionally arousing experiences. Annu. Rev. Neurosci., 27, 1-28.

McGaugh, J.L., Ferry, B., Vazdarjanova, A. \& Roozendaal, B. (2000) Amygdala: role in modulation of memory storage. In Aggleton, J. (ed.), The Amygdala: a Functional Analysis. Oxford University Press, New York, pp. 391-423.

O'Keefe, J. \& Bouma, H. (1969) Complex sensory properties of certain amygdala units in the freely moving cat. Exp. Neurol., 23, 384-398.

Paré, D. (2002) Mechanisms of Pavlovian fear conditioning: has the engram been located. Trends Neurosci., 25, 436-437.

Paré, D. \& Gaudreau, H. (1996) Projection cells and interneurons of the lateral and basolateral amygdala: distinct firing patterns and differential relation to theta and delta rhythms in conscious cats. J. Neurosci., 16, 3334-3350.

Pascoe, J.P. \& Kapp, B.S. (1985) Electrophysiological characteristics of amygdaloid central nucleus neurons during Pavlovian fear conditioning in the rabbit. Behav. Brain Res., 16, 117-133.

Paxinos, G. \& Watson, C. (1999) The Rat Brain in Stereotaxic Coordinates. Academic Press, San Diego, CA.

Pitkänen, A. (2000) Connectivity of the rat amygdala complex. In Aggleton, J. (ed.), The Amygdala: a Functional Analysis. Oxford University Press, pp. 31-115.

Poremba, A. \& Gabriel, M. (2001) Amygdalar efferents initiate auditory thalamic discriminative training-induced neuronal activity. J. Neurosci., 21, 270-278.

Quirk, G.J., Armony, J.L. \& LeDoux, J.E. (1997) Fear conditioning enhances different temporal components of toned-evoked spike trains in auditory cortex and lateral amygdala. Neuron, 19, 613-624.

Quirk, G.J., Repa, J.C. \& LeDoux, J.E. (1995) Fear conditioning enhances short-latency auditory responses of lateral amygdala neurons: parallel recording in the freely behaving rat. Neuron, 15, 1029-1039.

Repa, K.C., Muller, J., Apergis, J., Desrochers, T.M., Zhou, Y. \& LeDoux, J.E. (2001) Two different lateral amygdala cell populations contribute to the initiation and storage of memory. Nat. Neurosci., 4, 724-731.

Roozendaal, B., McReynolds, J.R. \& McGaugh, J.L. (2004) The basolateral amygdala interacts with the medial prefrontal cortex in regulating glucocorticoid effects on working memory impairment. J. Neurosci., 24, 1385-1392.

Rosat, R., Da-Silva, R.C., Zanatta, M.S., Medina, J.H. \& Izquierdo, I. (1992) Memory consolidation of a habituation task: role of N-methyl-D-aspartate, cholinergic muscarinic and GABA-A receptors in different brain regions. Braz. J. Med. Biol. Res., 25, 267-273.

Rosenkranz, J.A. \& Grace, A.A. (2002) Dopamine-mediated modulation of odour-evoked amygdala potentials during Pavlovian conditioning. Nature, 417, 282-287.

Schoenbaum, G., Chiba, A.A. \& Gallagher, M. (1999) Neural encoding in orbitofrontal cortex and basolateral amygdala during olfactory discrimination learning. J. Neurosci., 19, 1876-1884.

Setlow, B., Schoenbaum, G. \& Gallagher, M. (2003) Neural encoding in ventral striatum during olfactory discrimination learning. Neuron, 38, 625-636.

Tamásy, V., Korányi, L. \& Lissák, K. (1975) Multiple unit activity study of brain stem and limbic structures during environmental habituation and circadian rhythm. Pflugers Arch., 353, 361-372.

Tsai, M.L. \& Yen, C.Y. (2003) A simple method for fabricating horizontal and vertical microwire arrays. J. Neurosci. Meth., 131, 107-110.

Walker, D.L. \& Davis, M. (2002) The role of amygdala glutamate receptors in fear learning, fear-potentiated startle, and extinction. Pharmacol. Biochem. Behav., 71, 379-392.

Wolfman, C., Da Cunha, C., Jerusalinsky, D., Levi de Stein, M., Viola, H., Izquierdo, I. \& Medina, J.H. (1991) Habituation and inhibitory avoidance training alter brain regional levels of benzodiazepine-like molecules and are affected by intracerebral flumazenil microinjection. Brain Res., 548, 74-80.

Woof, N.J. \& Butcher, L.L. (1981) Cholinergic neurons in the caudate-putamen complex proper are intrinsically organized: a combined Evans blue and acetylcholinesterase analysis. Brain Res. Bull., 7, 487-507.

Zald, D.H. (2003) The human amygdala and the emotional evaluation of sensory stimuli. Brain Res. Rev., 41, 88-123. 